Freshwater Biological Association's key, 121 in Ward and Whipple's and 60 are common, but this is a group well known for its cosmopolitan distribution and in many others the overlap is much less.

The references vary from chapter to chapter; sometimes there are none to European work, sometimes the main ones are included, particularly if they are written in English.

It is clear, then, that whoever uses the new "Ward and Whipple" will have to struggle with keys that are larger than is necessary for his purpose and which probably do not include the species before him. Moreover, there is much more on the European fauna than was available to his predecessor using the first edition. On the other hand, almost all freshwater animals and plants are dealt with between two covers and the book must therefore contain some organism allied to any specimen the identity of which is to be ascertained. This can be comforting to somebody who has prowled in vain around a scientific library searching for a key to the group to which a specimen is thought to belong, and even more comforting to somebody who has no scientific library to prowl around. A second point that will endear this new volume to a new generation is the full treatment of neglected groups. I believe, therefore, that the book will be found not to have lost its old appeal.

T. T. MACAN

\section{OPTICAL ROTATORY POWERS OF ALKALOIDS}

Tables of Constants and Numerical Dita

11: Selected Constants: Optical Rotatiry Power. 4: Alkaloids. By Prof. J.-P. Mathieu and Prof. M.-M. Janot. (Publication subsidized by the Member Countries of the International Commission of $\%$ ables of Constants and by the Centre National de la Recherche Scientifique.) Pp. v+211. (London and New York : Pergamon Press, 1959.) 160s. net.

$7 \mathrm{HE}$ present volume is the eleventh in the series of "Tables of Constants and Numerical Data", founded in 1909 by C. Marie, and it is the fourth in the series devoted to the optical rotatory powers of naturally occurring substances. The preceding volumes dealing with optical rotatory power have covered the steroids (1956), the triterpenoids (1958), and the amino-acids (1959), and all four have come under the general editorship of Prof. J.-P. Mathieu with the aid of specialists in the particular fields concerned. In this volume, devoted to the alkaloids, Prof. M.-M. Janot has collaborated with Prof. Mathieu, and Sir Robert Robinson has provided a foreword.

In view of the greater chemical diversity of the alkaloids the material covered in this volume is inevitably more heterogeneous than that considered in the preceding volumes, and the main function of the book is to collect together in one place the data scattered throughout the literature on optical rotation measurements made on alkaloids. The editors have been faced with the well-known problem of defining an alkaloid, and they have therefore based their criteria for inclusion on the considerations that the alkaloids form a group of organic compounds of vegetable origin, always containing nitrogen, possessing more or less pronounced basicity, yielding precipitates with certain 'general' reagents, and being endowed with remarkable pharmacological activities. It is realized, of course, that such a classification is somewhat arbitrary, and the editors have compiled a list of the known alkaloids, including minor alkaloids and many that have no action on polarized light, provided that an empirical formula has been assigned.

In the table of rotatory powers, the alkaloids and their salts are listed in alphabetical order by trivial names with molecular (or empirical) formulæ and melting points. Other column entries are molecular weight, and solvent, concentration, temperature, and wave-length for which the recorded specific rotations have been measured, and, finally, bibliographic references. The table, which has been compiled essentially from data found in original papers, occupies 166 pages, and the bibliographic references, given in a most convenient and compact form, occupy 23 pages, followed by an author index (10 pages) and a formula index (8 pages). The references (1809) span the interval between 1873 and mid-way through 1959 , and their distribution over the years provides an interesting sidelight on activity in the field of alkaloid chemistry. Thus, the six decades from 1870 to 1929 provided only $19 \cdot 3$ per cent of the references, and the three decades since have provided respectively $18 \cdot 4$, $14 \cdot 7$, and no less than $47 \cdot 7$ per cent of the references. In fact, about one-third $(32 \cdot 8$ per cent $)$ of the bibliography relates to the last five and a half years covered, and it is obvious that, at this time of intense activity in the field of alkaloids, the present volume will be welcomed in laboratories where alkaloids are being studied.

It is a pleasure to note that a return has been made to a conventionally printed style, employing minute but very legible type, and the binding matches that of earlier volumes in the series.

J. WALKER

\section{A MYTHICAL SCIENCE}

\section{The Masks of God}

Primitive Mythology. By Joseph Campbell. Pp. viii +504. (London: Martin Secker and Warburg, Ltd., 1960.) 35s. net.

THE publishers claim that "The Masks of God" is "undoubtedly a landmark in its own field". Alas! Like so many landmarks it is also a folly.

The author's purpose is "the first sketch of a natural history of the gods and heroes, such as in its final form should include in its purview all divine beings. . . . For, as in the visible world of the vegetable and animal kingdoms, so also in the visionary world of the gods : there has been a history, an evolution, a series of mutations, governed by laws; and to show forth such laws is the proper aim of science" (p. 5). A new-born chick, says Dr. Campbell, will dart for cover when a hawk, or a model of a hawk, passes overhead-but not when the bird is a gull, duck, heron, or pigeon, or model of these latter. This kind of response to inherited, not taught, "innate releasing mechanisms", Dr. Campbell continues, is shared by human beings ; and the biology, psychology, sociology and history of the sign stimuli which release these mechanisms of inheritance "constitute the field of our subject, the science of Comparative Mythology" (p. 4l).

There is here a major distinction between what is common to all men and what is culturally determined; 